\title{
CORRESPONDENCE \\ DNA methylation profile of a hepatosplenic gamma/delta T-cell lymphoma patient associated with response to interferon- $\alpha$ therapy
}

\author{
Jaydeep Bhat $\mathbb{D}^{1,2}$, Anke K. Bergmann ${ }^{3,4}$, Silvio Waschina $\mathbb{D}^{5,6}$, Christoph Nerl ${ }^{7}$, Christoph Kaleta ${ }^{5}$, Reiner Siebert ${ }^{3,8}$ \\ Ole Ammerpohl ${ }^{3,8}$ and Dieter Kabelitz (iD) \\ Cellular \& Molecular Immunology (2021) 18:1332-1335; https://doi.org/10.1038/s41423-020-0518-4
}

The $2-5 \%$ $\gamma \delta \mathrm{T}$ cells in healthy human peripheral blood recognize nonpeptide antigens, in contrast to $a \beta T$ cells. ${ }^{1}$ Distinct $\gamma \delta$ T-cell malignancies have been identified, with hepatosplenic T-cell lymphoma $(\gamma \delta-H S T L)$ being a rare but aggressive (median survival 13 months) subset. ${ }^{2} \gamma \delta$-HSTL usually presents with hepatosplenomegaly without lymphadenopathy, anemia, and thrombocytopenia. It is histologically characterized by the involvement of the hepatic sinusoids, splenic red pulp and bone marrow interstitium, with or without leukemic presentation in peripheral blood. ${ }^{3}$ A standard treatment regimen is not well established, but therapy mostly includes various chemotherapeutic agents. Notably, interferon- $a$ (IFN- $a$ ) has been proven to be effective in some patients with $\gamma \delta$ HSTL, but other patients require allogeneic or autologous stem cell transplantation. ${ }^{4}$

Here, we present a longitudinal comprehensive DNA methylation analysis of a patient with $\gamma \delta$-HSTL who reached complete remission on continuous therapy with interferon-a2c (IFNa2c). The 39-year-old female patient presented with chronic lymphocytosis

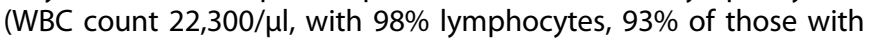
the $\gamma \delta$ T-cell clonal phenotype), mild hepatosplenomegaly, severe anemia (hemoglobin $(\mathrm{Hb})$ level $8.9 \mathrm{~g} / \mathrm{dL}$, haptoglobin level $<5 \mathrm{mg} /$ dl), $\mathrm{LDH} 300 \mathrm{U} / \mathrm{ml}$ and distinct Coombs-positive hemolysis. No peripheral, intrathoracic, or abdominal lymph node enlargements were recorded, but the skin showed multiple vitiligo-like lesions with lymphocyte infiltration. Bone marrow infiltration was also present. The expanded circulating lymphocytes resembled large granular lymphocytes (LGLs) with TCRy $\delta$ surface expression. ${ }^{5-7}$ The patient was treated with IFNa2c $\left(10^{6} \mathrm{IU}\right.$ s.c.) over a period of 6 years. During the first two years of treatment, an increase in the $\mathrm{Hb}$ level (from 8.9 to $11.2 \mathrm{~g} / \mathrm{dL}$ ) and a steady decrease in the leukocyte count (from $22,300 / \mu$ l to $7200 / \mu$ l) were observed. The hematological parameters and peripheral blood mononuclear cell (PBMC) phenotyping were identical before and after the visit at which IFNa2c treatment was initiated ${ }^{5}$ (Supplementary Table S1).

In this retrospective study using frozen PBMCs collected from this patient at five time points (hereafter referred to as visits 1-5) over 22 months after the initiation of IFNa2c therapy, DNA methylation analysis was performed using Infinium 450k BeadChip arrays (see Supplementary Material). After removing all measurements with detection $p$ values $>0.001$, we applied a variance filter $\left(\sigma / \sigma_{\max }>0.01\right)$ to focus on loci showing the strongest changes $(n=$ 12,825 loci). After performing a Pearson correlation analysis (day of treatment $\sim$ avg. beta value), we included only CpG loci that showed a significant (FDR $<0.05$; Benjamini-Hochberg) and continuous change in DNA methylation under treatment (day 54-652). Of the resulting 273 loci, 49 showed an increase in methylation (Pearson correlation coefficient range: $0.9908-0.9990)$, while the remaining loci showed a negative correlation (Pearson correlation coefficient range: -0.9907 to -0.9916$)$ (Supplementary Table S2).

In the exploratory analysis, we observed statistically significant (paired Wilcoxon test, FDR $<0.05$; Benjamini-Hochberg) changes in the overall DNA methylation of 273 CpGs (Fig. 1a, b, Supplementary Fig. S1) and correlated changes as described above (Fig. 1c). The principal component analysis (PCA) showed distinct visitdependent changes mainly due to PC1 $96.7 \%$ (Fig. 1d). Of note, the top genes, such as IFI44L (cg27315157), MEGF6 (cg06353486), MX1 (cg08080029), and CHD5 (cg26312951), contributed to 3.2\% of the PC2 distribution (Supplementary Table S2). Using the Illumina HumanMethylation450 annotation data (hg19), we analyzed the corresponding genes as well as the CGI and genomic features of each of the $273 \mathrm{CpG}$ sites (Supplementary Fig. S2A). A statistically significant change in DNA methylation was observed in a timedependent manner, represented here only for visit 5 and visit 1 , in the genomic regions (Supplementary Fig. S2B-C), viz. the $5^{\prime}$ UTR (adj. $p$ value $=0.0007$ ), gene body (adj. $p$ value $=8.81 \mathrm{e}-17$; probably due to the maximum number of $\mathrm{CpG}$ sites included in this group, $n=144$ loci) and $3^{\prime}$ UTR regions (adj. $p$ value $=0.012$ ).

Interestingly, the functional annotation of genes related to the 273 CpG sites was enriched for important pathways, such as proteoglycans in cancer (e.g., $C D K N 1 A)^{8}$ and the Wnt signaling pathway, and their regulatory transcription factors (Supplementary Fig. S3A), such as MZF1, SP1, and ARNT, and regulating biological processes, such as steroid hormone signaling and innate immune response (Fig. 1e, Supplementary Table S3), indeed reflect a

\footnotetext{
${ }^{1}$ Institute of Immunology, Christian-Albrechts-University Kiel \& University Hospital Schleswig-Holstein, Campus Kiel, Kiel, Germany; ${ }^{2}$ Metabolic Programming, School of Life

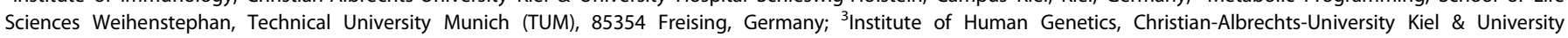

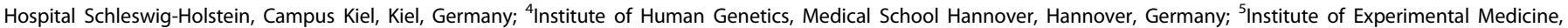

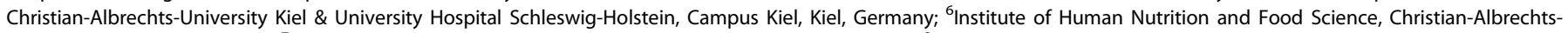

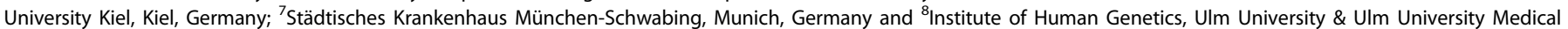
Center, 89081 Ulm, Germany

Correspondence: Ole Ammerpohl (ole.ammerpohl@uni-ulm.de) or Dieter Kabelitz (dietrich.kabelitz@uksh.de)
}

Received: 16 June 2020 Accepted: 16 July 2020

Published online: 20 August 2020 
A

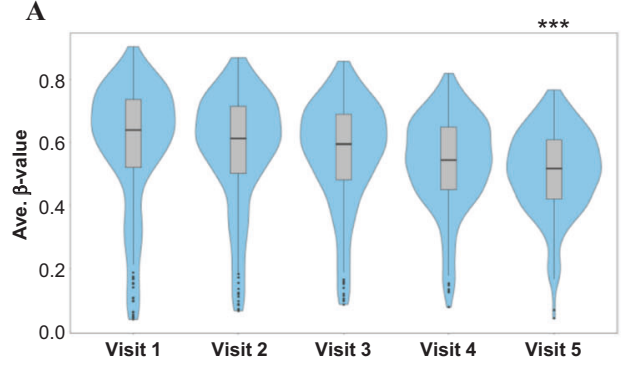

C

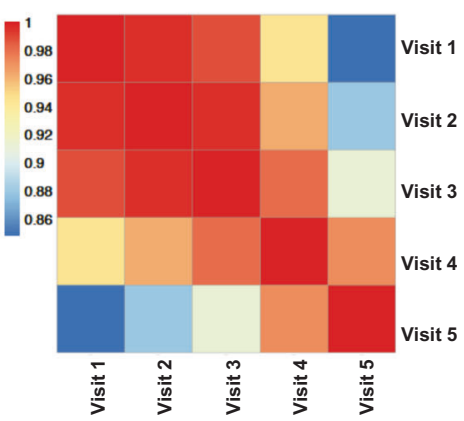

$\mathbf{E}$

Regulation of cilium assembly Neuroligin clustering [...] membrane assembly Response to mycotoxin Lung induction Innate immune response Adult behavior -

Cellular response to tumor necrosis factor Steroid hormone mediated signaling pathway

Positive regulation of [...] morphogenesis Cell adhesion Positive regulation of myelination Nervous system development

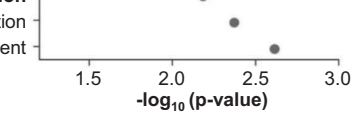

B

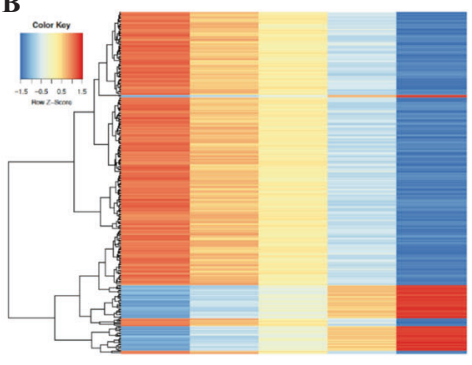

Visit 1 Visit 2 Visit 3 Visit 4 Visit 5

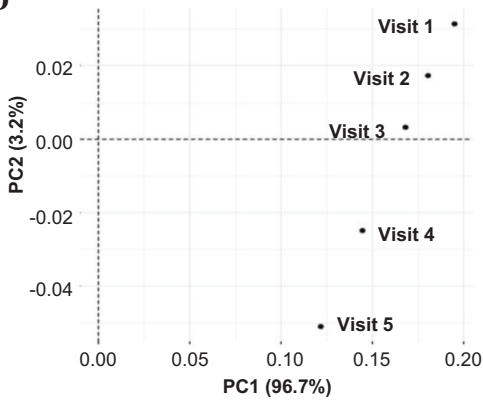

Cellular component

$$
\text { Collagen trimer } \mid \text {. }
$$
$Z$ disc

Proteinaceous extracellular matrix

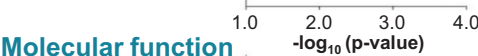
.

teroid hormone receptor activity

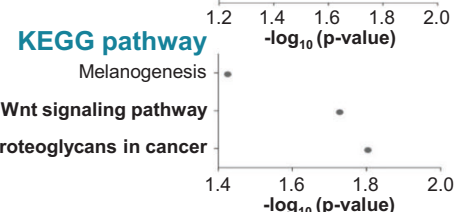

G

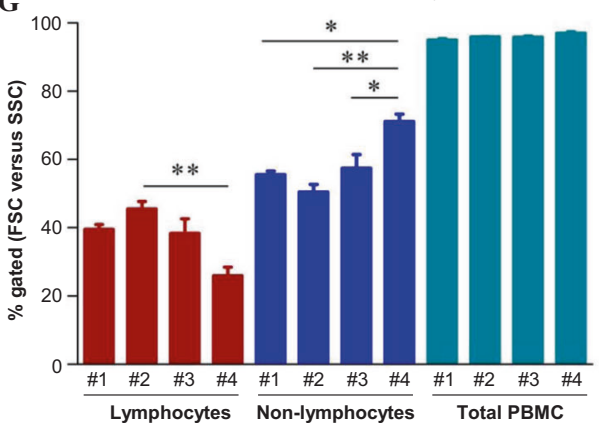

Fig. 1 DNA methylation analysis of the $\gamma \delta$-HSTL patient during IFN $\alpha 2 c$ therapy. DNA methylation analysis was performed on bisulfite-converted DNA from PBMCs of the $\gamma \delta$-HSTL patient obtained at five different time points during continued IFN $\alpha 2 \mathrm{c}$ therapy. a The violin plot represents the distribution of the methylation of 273 statistically significant $\mathrm{CpG}$ loci at different visits, including the quartiles and median methylation. The DNA methylation level was calculated using the average $\beta$-value of the respective CpG site. Only for statistical purposes was the adjusted $p$ value calculated for visits 1 and 5 using the paired Wilcoxon test. The statistical significance is shown as *** for $p$ values $\leq 0.005$. $\mathbf{b}$ The heatmap was plotted after normalization to the mean methylation level (average $\beta$-value) of CpG sites, as indicated by scales in the color key. c The heatmap presents Pearson's correlation values from visit 1 to visit 5 . The range of correlation values is shown in the color key at the top-left side of the plot. d The principal component analysis (PCA) plot of significant CpG loci (similar to the violin plot) represents the separation of two different components depending on the DNA methylome during the visits of the $\gamma \delta$-HSTL patient. e The functional gene ontology (GO) analysis of genes related to the CpG loci from visits 1 to 5 . The GO terms enriched in categories, namely, biological process (left-side), cellular component (right-side top), molecular function (right-side middle), and KEGG pathway (right-side lower), are presented for criteria of statistical significance $p$ value $\leq 0.05$. f The cosine similarity score was calculated as described in the Supplementary material. Cosine similarity between the DNA methylation of healthy T cells and cells from the $\gamma \delta$-HSTL patient at visits (1-5) during IFN $\alpha 2 c$ therapy. The statistical significance of $p$ values $\leq 0.05$ is represented as *** for the difference between visit 1 and healthy T cells. $\mathbf{g}$ As shown in Table S1 and represented in Supplementary Fig. 4B, flow cytometry analysis of PBMCs in $\gamma \delta$-HSTL patient visits during IFN $\alpha 2 c$ therapy was performed. Based on forward scatter (FSC) and sideward scatter (SSC), lymphocytes and non-lymphocytes were gated within the PBMCs. The data are represented as the percentage gated with a statistically significant difference for $p$ values $\leq 0.05, \leq 0.01$, and $\leq 0.005$ shown as ${ }^{* * *}$, ${ }^{* *}$, and ${ }^{*}$, respectively 
possible relation of the multiple epigenetic changes with the described clinical features. In addition to the 273 loci retained from the data analysis detailed above, the DNA methylation level of cg26960322 (related to the 5'UTR region of the CXCR7 gene; an immunologically important gene as well as a potential target for cancer therapy, as it modulates cellular proliferation, survival and migration in the bone marrow and lymphoid organs) ${ }^{9}$ and the WBC counts at the different time points correlated well ( $p$ value $=$ 0.0167), suggesting a possible functional relevance of regulatory changes in DNA methylation in this $\gamma \delta-H S T L$ patient during IFNa2c therapy (Supplementary Fig. S4A).

In a previous report, using the same material (data from visit 1 only) from this $\gamma \delta$-HSTL patient (sample "1_Kab17"), the DNA methylation patterns of HSTL patients, including both $a \beta$ - and $\gamma \delta$ HSTL, were compared to those of healthy individuals' a $\beta$ - and $\gamma \delta-T$ cells. In this study, altered DNA methylation was observed in HSTL patients exhibiting the hypermethylation of key genes at the active promoter and regulatory enhancer regions. ${ }^{7}$ The $\gamma \delta$-HSTL patient studied in our present longitudinal study carried a STAT3 mutation ${ }^{7}$; thus, IFNa2c can induce proapoptotic gene expression. ${ }^{10}$ However, clonal $\gamma \delta$ T cells at visit 1 were refractory to the in vitro treatment of IFNa and lacked proliferative and cytotoxic capacity. ${ }^{5}$ Since the $\gamma \delta$ Tcell phenotype of PBMC blasts of this $\gamma \delta$-HSTL patient remained the same (87-93\% of lymphocytes), with a steady decrease in WBC/blast counts leading to complete clinical remission after IFNa2c therapy, we used a cosine similarity score model to evaluate the similarity prediction between the DNA methylation profile of the cells from the $\gamma \delta$-HSTL patient as a result of IFNa2c therapy and that of healthy $T$ cells. This computational approach (Fig. 1f), in support of flow cytometry analysis (Fig. 1g, Supplementary Fig. S4B) and clinical data (Supplementary Table S1), revealed that the DNA methylation profile of this patient in response to IFNa2c treatment (leading to clinical remission) was mainly associated with the non-lymphoid compartment of PBMCs and/or IFNa2c-responsive blasts. This observation was also supported by opposing DNA methylation levels of visit 5 and healthy T cells (Supplementary Fig. S3B). Though it is of great interest, it remains still unclear at this point what could be the functional epigenetic status of PBMCs after complete remission is reached, i.e., 6 years after the initiation of therapy.

DNA methylation profiling of the $\gamma \delta$-HSTL patient during the course of IFNa2c therapy in such a comprehensive but in-depth setting has shed light on important biological aspects in terms of (i) the protein regulation of the extracellular matrix and its relevance to cancer progression (e.g., ADAMTS16), ${ }^{11}$ (ii) the direct effect of IFNa2c on interferon hallmark gene regulation (e.g., CDKN1A, MX1), ${ }^{12}$ (iii) the effect of steroids used at the beginning of IFNa2c therapy and the involvement of nuclear receptors important for diverse metabolic functions (e.g., NR1/3), ${ }^{13-15}$ and (iv) the change in the (non-) lymphoid compartment in the PBMCs of the $\gamma \delta$-HSTL patient (e.g., LCN2), ${ }^{16,17}$ as shown before for the effect of steroids. ${ }^{18}$ To the best of our knowledge, this is the first case report exploring the effect of IFNa2c therapy on DNA methylation and possibly other regulatory epigenetic elements in $\gamma \delta$-HSTL. Based on this retrospective study of a single $\gamma \delta$-HSTL patient, we propose a complex remodeling of the epigenome during IFNa2c therapy, which achieves remission and a clinically healthy state. Though the results of this case report need to be verified in more patients, we provide an in-depth analysis of the IFNa2c therapy-induced changes in CpG methylation, which might serve as potential biomarkers of drug responses and/or disease progression.

\section{ACKNOWLEDGEMENTS}

We thank Monika Kunz, Pradeep Dagur and Manikandan Narayanan for their excellent technical support. We appreciate valuable and critical comments on the manuscript from Martin Vingron. We are very grateful to Florian Schmidt, Daniela Esser, and Marcel Schulz for help with the bioinformatics analysis using the cosine similarity predictions. This work was supported by the Deutsche Forschungsgemeinschaft (DFG) through the Cluster of Excellence "Inflammation-at-Interfaces" EXC306-PN and EXC306-PM (D.K., O.A. and C.K.) and the Werner-und-Klara-Kreitz Foundation (JB).

\section{AUTHOR CONTRIBUTIONS}

J.B., O.A., and D.K. designed the study; J.B. and S.W. performed the experiments; J.B., O.A., S.W., and D.K. analyzed the data; A.K.B. and R.S. supported the data interpretation; C.N. provided the patient material and clinical data; and J.B., C.K., O.A., and D.K. interpreted and discussed the data and wrote the manuscript. All authors approved the final draft of the manuscript.

\section{ADDITIONAL INFORMATION}

The online version of this article (https://doi.org/10.1038/s41423-020-0518-4) contains supplementary material.

Competing interests: The authors declare no competing interests.

\section{REFERENCES}

1. Vantourout, P. \& Hayday, A. Six-of-the-best: unique contributions of gamma delta T cells to immunology. Nat. Rev. Immunol. 13, 88-100 (2013).

2. Swerdlow, S. H. et al. The 2016 revision of the World Health Organization classification of lymphoid neoplasms. Blood 127, 2375-2390 (2016).

3. Yabe, M., Miranda, R. N. \& Medeiros, L. J. Hepatosplenic T-cell Lymphoma: a review of clinicopathologic features, pathogenesis, and prognostic factors. Hum. Pathol. 74, 5-16 (2018).

4. Ferreri, A. J., Govi, S. \& Pileri, S. A. Hepatosplenic gamma-delta T-cell lymphoma. Crit. Rev. Oncol./Hematol. 83, 283-292 (2012).

5. Metzger, R., Heckl-Ostreicher, B., Nerl, C., Schondelmaier, S. \& Kabelitz, D. Immunological studies of gamma delta $\mathrm{T}$ cells in a case of large granular lymphocyte (LGL) leukemia: leukemic gamma delta $+\mathrm{T}$ cells are resistant to growth stimulation in vitro but respond to interferon-alpha treatment in vivo. Leuk. Res. 16, 1087-1095 (1992).

6. Hinz, T., Marx, S., Nerl, C. \& Kabelitz, D. Clonal expansion of gamma delta T cells expressing two distinct T-cell receptors. Br. J. Haematol. 94, 62-64 (1996).

7. Bergmann, A. K. et al. DNA methylation profiling identifies candidate genes for the pathogenesis of hepatosplenic T-cell lymphoma. Haematologica 104, e104-e107 (2019).

8. lozzo, R. V. et al. Cooperative action of germ-line mutations in decorin and p53 accelerates lymphoma tumorigenesis. Proc. Natl. Acad. Sci. USA 96, 3092-3097 (1999).

9. Barbieri, F., Bajetto, A., Thellung, S., Wurth, R. \& Florio, T. Drug design strategies focusing on the CXCR4/CXCR7/CXCL12 pathway in leukemia and lymphoma. Expert Opin. Drug Discov. 11, 1093-1109 (2016).

10. Kiladjian, J. J., Mesa, R. A. \& Hoffman, R. The renaissance of interferon therapy for the treatment of myeloid malignancies. Blood 117, 4706-4715 (2011).

11. Kordowski, F. et al. Aberrant DNA methylation of ADAMTS16 in colorectal and other epithelial cancers. BMC Cancer 18, 796 (2018).

12. Kollmann, S. et al. Twins with different personalities: STAT5B-but not STAT5A-has a key role in BCR/ABL-induced leukemia. Leukemia 33, 1583-1597 (2019).

13. Hedrich, W. D. et al. Activation of the constitutive androstane receptor increases the therapeutic index of CHOP in lymphoma Treatment. Mol. Cancer Therapeutics 15, 392-401 (2016).

14. Yao, S. et al. Impact of chemotherapy for breast cancer on leukocyte DNA methylation landscape and cognitive function: a prospective study. Clin. Epigenetics 11, 45 (2019).

15. Wan, E. S. et al. Systemic steroid exposure is associated with differential methylation in chronic obstructive pulmonary disease. Am. J. Respir. Crit. Care Med. 186, 1248-1255 (2012).

16. Chakraborty, S., Kaur, S., Guha, S. \& Batra, S. K. The multifaceted roles of neutrophil gelatinase associated lipocalin (NGAL) in inflammation and cancer. Biochimica et. Biophysica Acta. 1826, 129-169 (2012).

17. Schroll, A. et al. Lipocalin-2 ameliorates granulocyte functionality. Eur. J. Immunol. 42, 3346-3357 (2012).

18. Chitadze, G. et al. In-depth immunophenotyping of patients with glioblastoma multiforme: Impact of steroid treatment. Oncoimmunology 6, e1358839 (2017). 
DNA methylation profile of a hepatosplenic gamma/delta T-cell lymphoma... $J$ Bhat et al.

Open Access This article is licensed under a Creative Commons acc Attribution 4.0 International License, which permits use, sharing, appropriate credit to the original author(s) and the source, provide a link to the Creative Commons license, and indicate if changes were made. The images or other third party material in this article are included in the article's Creative Commons license, unless indicated otherwise in a credit line to the material. If material is not included in the article's Creative Commons license and your intended use is not permitted by statutory regulation or exceeds the permitted use, you will need to obtain permission directly from the copyright holder. To view a copy of this license, visit http://creativecommons. org/licenses/by/4.0/.

(c) The Author(s) 2020 